\title{
Reduction of apoptosis and proliferation in endometriosis
}

Aude Béliard, M.D., Ph.D., Agnès Noël, Ph.D., and Jean-Michel Foidart, M.D., Ph.D.

Laboratory of Tumor and Development Biology, University of Liège, Liège, Belgium

Objective: To evaluate whether endometriosis could be related to an impaired balance between apoptosis and proliferation, two processes which could be modulated by hormonal status.

Design: Immunohistochemical study.

Setting: Academic research laboratory.

Intervention(s): Endometriotic samples obtained from peritoneum of women aged 26-40 years who were undergoing laparoscopy for pain or infertility.

Main Outcome Measure(s): Apoptotic cells were detected with the use of the terminal deoxynucleotidyl transferase-mediated dUTP nick-end labeling (TUNEL) assay. The production of p53 and bcl-2, estrogen and Progesterone (P) receptors, and cellular proliferation were assessed by immunohistochemistry in eutopic and ectopic endometria from 30 patients with endometriosis throughout the menstrual cycle. Results were compared with those from normal endometria from 15 fertile patients.

Result(s): Endometriotic lesions were characterized by reduced TUNEL and p53 stainings and by enhanced bcl2 staining. No correlation between apoptosis and estrogen receptor or P receptor levels was found. A lower amount of steroid receptor was found in endometriotic tissues, without cyclic modulation, compared with the eutopic endometrium.

Conclusion(s): Our results suggest that when endometrial tissue is located at ectopic locations, it differs from eutopic endometrium by its proliferation rate, steroid hormone levels, and markers of apoptosis. A reduced sensitivity of endometriotic cells to apoptosis could promote the dissemination and implantation of these cells to ectopic sites.

Key Words: Endometriosis, cellular proliferation, apoptosis, estrogen receptor, progesterone receptor

Endometriosis is a pathologic condition that involves abnormal endometrial cell adhesion, growth, and proliferation in ectopic tissues, such as the ovary and the peritoneal surface (1-2). The "decision" of cells to undergo apoptosis is controlled by external signals together with an autonomous genetic program. Several proteins, including bcl-2 and p53, regulate this complex process. The p53 and bcl-2 proteins can be regarded as positive and negative regulators of cell death, respectively (3).

Endometrium is divided into the superficia-lis or functionalis layer, which undergoes cyclic shedding, and the basalis layer, which is permanent. Endometrial tissue from the functionalis is subjected to a proliferative process highly regulated by hormones throughout the menstrual cycle. At the time of menstruation, it becomes necrotic and hypoxic and is shed. In addition, apoptosis seems to be an important biologic process involved in the cyclic remodeling of the endometrium (4). It is well known that menstrual fragments are composed of both necrotic and living cells (5-6).

In endometriosis, abnormal adhesion of endometrial fragments to ectopic sites could be due to an increase in the adhesive and proliferative properties of menstrual endometrium, a decrease in apoptosis, and/or anomalies in the target tissue microenvironment. The properties of floating endometrial cells that reach the peritoneal cavity are under the influence of the peritoneal fluid, which is known to be proinflammatory in women with endometriosis (7). 
In contrast, the endometrium is regulated by blood factors (8). In addition, ectopic endometrial cell implantation could be related to modification in apoptosis sensitivity. Indeed, under normal conditions, apoptosis is thought to prevent the dissemination and attachment of differentiated epithelial cells at inappropriate locations when they lose contact with matrix (9-10).

To evaluate the possibility that the balance between proliferation and apoptosis could be impaired and hormonally regulated in endometriosis, we evaluated cell proliferation, steroid hormone receptor levels, and apoptosis on biopsies of human endometrium and endometriosis.

The presence of proliferating cell nuclear antigen (PCNA) (a 36-kd nuclear protein restricted to S phase) and markers of apoptosis (DNA fragmentation, p53, and bcl-2) were assessed by immunohistochemistry in peritoneal endometriosis and in the corresponding endometrium throughout the menstrual cycle and compared with the endometrium from women without endometriosis (11).

\section{MATERIALS AND METHODS}

\section{Antibodies}

Monoclonal mouse anti-PCNA, monoclonal mouse anti-human bcl-2 oncoprotein antibody, and anti-p53 protein antibody were from Dako (Glostrup, Denmark), and antihuman estrogen receptor and antihuman P receptor antibodies were from Novocastra (Newcastle, United Kingdom).

\section{Endometrial Samples}

The institutional review board of the University of Liege (Belgium) approved the collection and use of human tissue. Normal human endometrium was obtained from nonmeno-pausal fertile women aged 30-45 years undergoing hysterectomy for benign gynecologic indications (subserosal leiomyomata) or laparoscopy for elective tubal ligation and who did not receive any hormonal treatment.

None of these patients had identifiable endometriosis. Endometrial dating was performed according to the method of Noyes et al. (12). Endometrial samples were obtained throughout the menstrual cycle (five were in the proliferative phase, five in the early secretory phase, and five in the late secretory phase).

Typical endometriotic lesions were removed from the peritoneum of the pouch of Douglas, the broad ligament, or the uterosacral ligament of women aged 26-40 years who were undergoing laparoscopy for pain (10 patients) or infertility (20 patients). Simultaneously, eutopic endometrium was obtained from the same women (10 were in the proliferative phase, 10 in the early secretory phase, and 10 in the late secretory phase).

To distinguish between endometrium from endometriotic and nonendometriotic patients, samples from nonendometri-otic patients were termed normal endometrium, and samples from endometriotic patients were termed eutopic endometrium.

\section{Immunohistochemistry}

Endometrium and endometriosis lesions were fixed in neutral buffered 10\% formaldehyde for 1 hour. In sections of $5 \mu \mathrm{m}$, histologic examination confirmed the presence of ectopic endometrial glands surrounded by stromal cells. Classic immunoperoxidase staining with monoclonal mouse antihuman bcl-2, antihuman p53, antihuman estrogen receptor (ER), and antihuman P receptor (PR) were performed. Protein expression was detected with a streptavidin-biotin peroxidase complex.

Briefly, after blocking endogenous peroxidase activity, sections were microwaved in citrate buffer (pH 6) for antigen retrieval. Slides were then washed in phosphate-buffered saline, incubated in normal swine serum (NSS) for 30 minutes, and then with anti-bcl-2 diluted 1:40 in NSS, anti-p53 diluted 1:25 in NSS for 1 hour at room temperature, anti-PR and anti-ER (overnight at $4^{\circ} \mathrm{C}$ ), and with horseradish perox-idase-conjugated anti-PCNA. Finally, sections were incubated with biotinylated antirabbit and antimouse immunoglobulins and with streptavidin peroxidase (LSAB kit; Dako, Glostrup, Denmark). The reaction was developed with 3-3' diaminobenzidine and hydrogen peroxide.

The slides were counterstained with hematoxylin. Negative controls were performed by replacing the primary antibody with normal serum. 


\section{Apoptosis Detection System}

Apoptosis was assessed with the principle of terminal deoxynucleotidyl transferase-mediated dUTP nick-end labeling (TUNEL) to detect fragmented DNA in apoptotic cells. Sections were treated according to the manufacturer's recommendations. Briefly, sections were deparaffinized and rehydrated, permeabilized in proteinase $\mathrm{K}$, and treated with terminal deoxynucleotidyl transferase incubation buffer at $37^{\circ} \mathrm{C}$ for 60 minutes. Sections were then treated with per-oxidase-labeled antidigoxigenin antibody, followed by 3'-3 diaminobenzidine color reaction. Sections were counterstained with hematoxylin.

\section{Quantification}

Evaluation of the nuclear staining for TUNEL-positive cells, p53, ER, PR, and PCNA was performed with a computer-assisted cellular imaging system (CAS 200; Bacus Laboratories, Lombard, IL). First, the nuclear threshold of cells was determined to measure the nucleus size. Then, the antibody threshold was assessed to distinguish positive nuclei from negative nuclei. Finally, the software calculated the percentage of positive cells from the total number of cells evaluated. We performed a separate quantification of positive stromal and glandular epithelial cells in endometrium and endometriosis.

This software provided an accurate, objective, and quantitative microscope-based method for the assessment of nuclear staining in tissue sections.

Bcl-2 is a membrane protein, and the staining after immunohistochemistry was distributed around the circumference of cells. We performed a semiquantitative evaluation of the staining in epithelial and stromal cells: the intensity of the staining was scored as $0=$ no staining; 1 = weak staining; 2 = moderate staining; and 3 = strong staining.

\section{Statistical Analysis}

The Pearson test was used to evaluate the correlation between steroid receptors and apoptosis. The Fisher test was used to analyze the contingency of the results; $P<.05$ was considered significant.

\section{RESULTS}

\section{Cell Proliferation in Normal Endometrium, Eutopic Endometrium, and in Endometriosis}

In endometrium, the maximal proliferative activity was found in glandular epithelium in the preovulatory phase. This proliferation rate decreased continuously during the secretory phase until the midluteal phase, for which no more proliferation was detected $(P<.0001)$ (Fig. 1A). In the stroma, the proliferation index was higher in the proliferative phase than in the early and late secretory phases $(P<.0001$ between proliferative phase and early secretory phase and $P=.0002$ between proliferative phase and late secretory phase; data not shown).

No significant difference in cell proliferation in eutopic endometrium was seen between endometriotic and nonendometriotic patients.

In endometriotic lesions, the proliferative activity was significantly lower in the proliferative phase of the cycle compared with the endometrium and remained essentially constant throughout the cycle $(P=.3$ between proliferative phase and early secretory phase and $P=.1$ between early secretory phase and late secretory phase).

\section{ER and PR in Normal Endometrium, Eutopic Endometrium, and in Endometriosis}

In normal and eutopic endometrium, the percentage of ER- and PR-stained nuclei was high in epithelial and stromal cells in the proliferative phase and low in the secretory phase (Fig. 1B and C).

In endometriotic lesions, hormone receptors did not exhibit the cyclic variation occurring in normal and eutopic endometrium. Estrogen receptor levels were low throughout the cycle in endometriosis (nuclear staining intensity <10\%). Progesterone receptor levels were stable throughout the cycle in endometriosis: nuclear staining intensity ranged from $14 \% \pm 5 \%$ (mean \pm SD) in the proliferative phase to $17 \% \pm 3.5 \%$ in the late secretory phase. In normal and eutopic endometrium, the PR levels were $3 \% \pm 0.5 \%$ in the late secretory phase $(P<.05$ compared with endometriosis). 


\section{p53, bcl-2, and TUNEL in Normal Endometrium, Eutopic Endometrium, and in Endometriosis}

In normal and eutopic endometrium, p53, bcl-2, and TUNEL showed cyclic changes. Deoxyribonucleic acid cleavage was seen at low levels in the proliferative phase, was almost absent in the early secretory phase, and was at a high level in the late secretory phase (Fig. 2). p53 was essentially detected in the late secretory phase, with marginal differences between the three considered tissues (percentage of positive nuclei: 0 in the proliferative phase for endometrium and endometriosis; $15 \% \pm 1.3 \%$ in endometrium; and $7 \% \pm 2.2 \%$ in endometriosis in the late secretory phase). Conversely, bcl-2 was highly expressed in the proliferative phase in normal and eutopic endometrium, whereas it was not detected in these tissues during the early and late secretory phases.

Figure1. Quantification of staining (percentage of positive cells) of eutopic endometrium and endometriosis from proliferative (dark blue bars), early secretory (open bars), and late secretory (light blue bars) phases with (A) monoclonal mouse an-tihuman anti-PCNA antibody, (B) antihuman anti-ER antibody, and (C) antihuman anti-PR antibody. The results are presented as the mean \pm SD of a minimum of 1,000 nuclei. Normal endometrium = endometrium from normal fertile patients without endometriosis; eutopic endometrium $=$ endometrium from endometriotic patients.

(A)

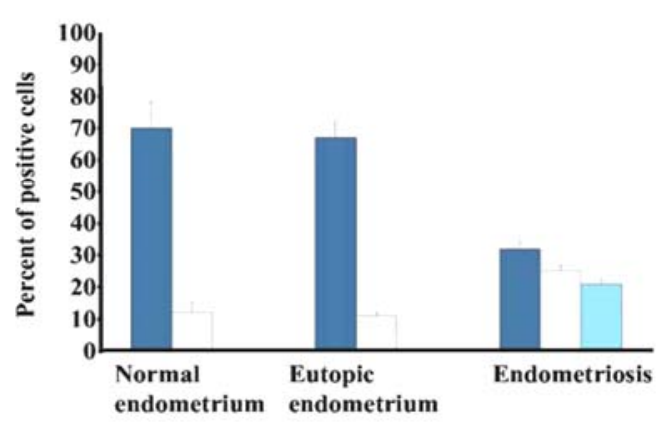

Tissue

(B)

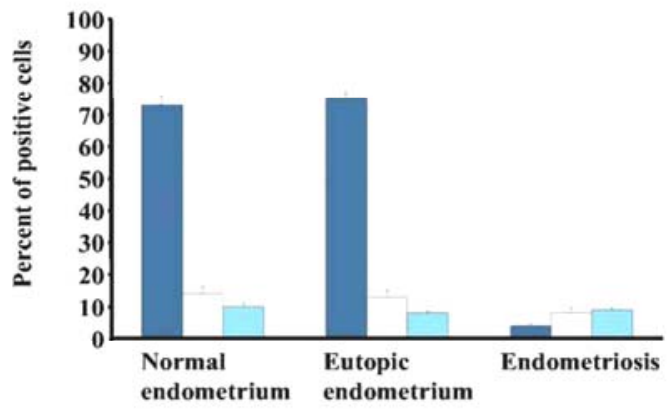

Tissue

(C)

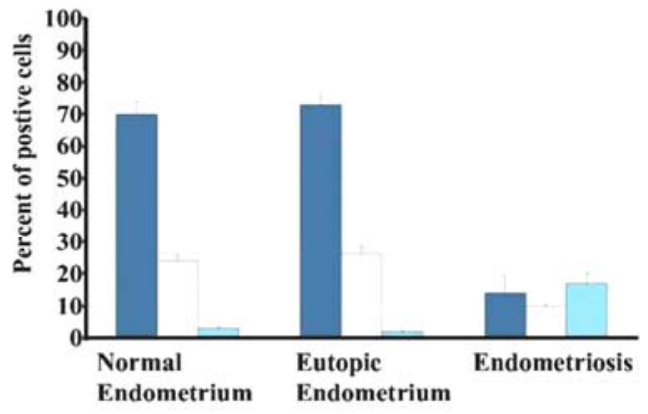

Tissue 
Figure2. Quantification of staining (percentage of positive cells) of eutopic endometrium and endometriosis from proliferative (dark blue bars), early secretory (open bars), and late secretory (light blue bars) phases with TUNEL assay with immu-noperoxidase staining. The results are presented as the mean \pm SD of a minimum of 1,000 nuclei. Normal endometrium = endometrium from normal fertile patients without endometriosis; eutopic endometrium = endometrium from endometriotic patients.

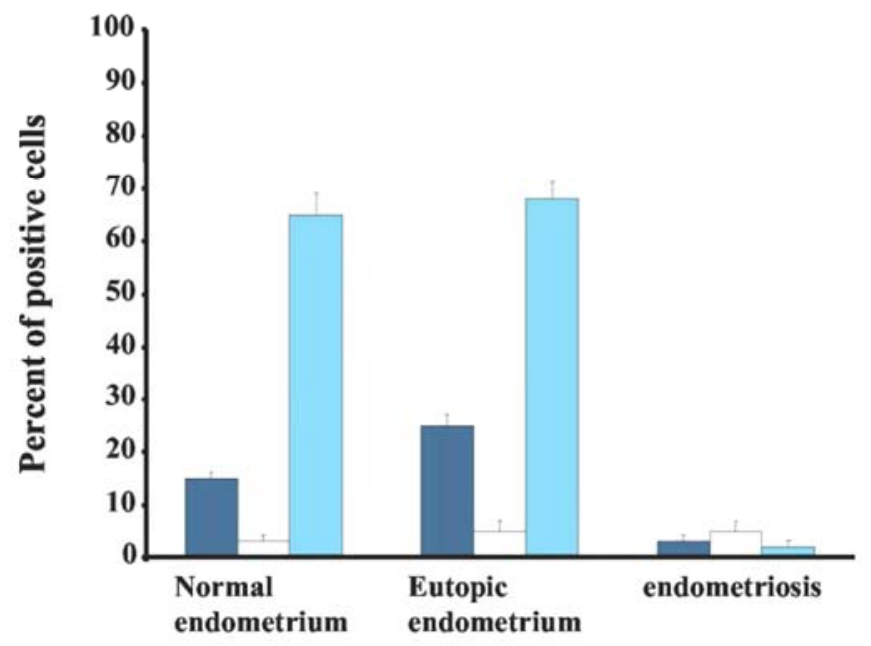

Tissue

In endometriosis, the level of DNA fragmentation was very low throughout the menstrual cycle. Although bcl-2 was found regardless of the cycle phase, its levels were high in the proliferative and late secretory phases and low in the early secretory phase.

In endometrium (normal and eutopic), there was a positive correlation between proliferation and ER levels $(P<.0001$ and $r=0.9448$ for proliferative phase) and an inverse correlation between proliferation ER levels and ap-optosis $(P<.0001$ and $r=-0.8660$ for the proliferative phase and $P<.0001$ and $r=-0.8852$ for the late secretory phase). Proliferation and ER levels were low during the late secretory phase, whereas apoptosis was high. No significant correlation was found between proliferation ER, PR, and DNA cleavage of glandular and stromal endometriotic cells $(P>.05$ and squared $=40.50 \%)$.

\section{DISCUSSION}

Although retrograde menstruation is a common phenomenon in women, it does not result in endometriosis in all women. This suggests that retrograde menstruation might promote endometrial tissue dissemination in the peritoneal cavity but that some other factors are required for the implantation and growth of ectopic endometrium. These additional factors could be an impaired peritoneal environment (cytokines, chemokines, Inflammatory cells) or alteration of the peritoneal lining or specific features of the endometrium itself (13).

It is likely that in healthy women, endometrial cells and tissue fragments expelled during menses do not survive in ectopic locations because of programmed cell death. Indeed, in normal tissue, epithelial cells require interactions with the extracellular matrix to survive and grow. Disruption of cell-matrix interactions induce cell death through a process called anoikis (10). This might prevent detached normal cells from implanting in a new and inappropriate location. A decrease in apoptosis might lead to ectopic survival and implantation of endometriotic cells and development of endometriosis.

Different studies have been performed to evaluate apoptosis in endometriosis and endometrium. Apoptotic cells were detected with an ELISA-based cell death detection kit (14) or the TUNEL assay for DNA fragmentation (15). To evaluate cell death repressor activity, bcl-2 was also determined by immunohistochemistry (16-20). To the best of our knowledge, no study has yet evaluated the proliferation rate and steroid hormone receptor levels in parallel with apoptosis assessment. Proliferative properties have been determined in combination with apoptosis in adenomyosis but not in endometriosis (21).

The present study combined for the first time an evaluation of markers of apoptosis, proliferation, and steroid hormone receptors in endometrial and endometriotic cells. 
The main interesting finding was a lower sensitivity of endometriotic cells to apoptosis. Controversial data regarding the apoptotic index in endometriosis vs. endometrium have been reported: bcl-2 was negative in almost all samples from ovarian endometriosis $(18,20)$, but bcl-2 was significantly increased in another study (16); apoptosis was increased in ovarian endometriosis (20) but decreased in single-cell suspensions of ectopic endometrium (14).

The comparison of results obtained in different studies is hampered by the use of different methodologies and different endometriotic lesions (ovarian vs. peritoneal endometriosis). Ovarian and peritoneal endometrioses are thought to have different pathogenetic origins. Ovarian endometriotic cysts could arise from invagination and metaplasia of ovarian epithelium lining, and peritoneal endometriosis is thought to be the consequence of implantation of shed endometrium (22).

Therefore, ovarian endometriosis could have different biological features. In ovarian endometriosis and not in peritoneal endometriosis, apoptosis was greater compared with eutopic endometrium $(18,20)$.

Some studies compared the apoptotic level in endometriosis with that in endometrium from the same patients; other studies also considered the levels of apoptosis in endometrium from normal fertile women. In these studies, as in our study, apoptosis was impaired in endometriosis, and no cyclic change was demonstrated $(14,16,17)$.

Finally, some authors used endometrium from infertile patients without endometriosis as a control. However, endometrium from infertile women can not be used as a control because such endometrium can be considered abnormal in terms of cell adhesion receptors $(23,24)$ and microscopic peritoneal endometriosis $(26,27)$. Therefore, endometrium from infertile women could not be used as a control for comparison with endometrium from endometriotic patients.

To overcome these problems, we analyzed both apoptotic and proliferative parameters in endometrium from normal fertile patients (control endometrium) and in endometriotic patients (eutopic endometrium).

No difference was demonstrated between endometrium from endometriotic vs. nonendometriotic women. This is consistent with the report of Jones et al. (16), in which no significant differences in apoptotic cell rate were found in endometrium from endometriotic and nonendometriotic women. In contrast, a decreased susceptibility to spontaneous apoptosis of endometrial tissue from endometriotic patients was described when compared with fertile control subjects (14).

In this study, apoptosis was evaluated in single endometrial cell suspensions after enzymatic digestion. However, it should be noted that the properties of monodispersed cells could be different from those of tissues in which paracrine interactions occur between epithelial and stromal cells or between endometriotic cells and the peritoneal micro-environment.

Other experiments also reported decreased apoptosis in eutopic endometrium from women with endometriosis compared with endometrium from control women (38). Although these investigators suggested a regulation of apoptosis and bcl-2 by ovarian steroids, ER and PR were not evaluated. In our study, although no difference in apoptosis was detected between endometrium from normal patients and endometrium from patients with endometriosis, the most interesting finding is the reduction of apoptosis in endometriotic lesions.

Steroid receptors were evaluated in parallel with apoptotic markers, and no correlation was found between the level of ovarian receptors and apoptosis in endometriosis. Apoptosis was not correlated with phases of the menstrual cycle in endometriosis, as in endometrium. Clinical studies (28) have suggested that in women with endometriosis, misplaced endometrial cells implanted in ectopic locations can lead to endometriotic lesions, whereas in healthy women such implantation does not succeed.

Our study indicates that because there was no difference in spontaneous apoptosis between endometrium from endometriotic and nonendometriotic patients, ectopic implantation of endometrium could not be ascribed to a decrease in apoptosis in endometrium. These results are in agreement with in vitro and animal studies using endometrium from women without endometriosis in their model of ectopic implantation and showing peritoneal adhesion of such normal endometrium (29-32). Normal endometrium from patients without endometriosis had, therefore, the ability to implant at ectopic locations.

Endometriotic lesions showed an increase in bcl-2 expression compared with endometrium. Such an elevated bcl-2 expression could promote the survival of endometriotic tissue at ectopic sites. These results are consistent 
with previous reports (16). Endometriotic cells, like tumor cells, could gain growth advantage by blocking the apoptotic pathway and stimulating cell proliferation.

Endometriotic cells, however, showed no enhanced proliferative properties, unlike tumor cells, which develop uncontrolled proliferation (33). Endometriosis could not be considered a mere graft of endometrial tissue with the same phenotypic characteristics: endometrium implanted at ectopic locations exists under different biologic properties than endometrium at eutopic locations. The transformation of the ectopic tissue could be the consequence of local factors in the peritoneal environment.

In agreement with other studies, no difference of proliferation rate was seen in endometrium from patients with vs. without endometriosis (34). Interestingly, significantly reduced proliferation activity was observed in endometriosis compared with endometrium. That could relate to the lower ER status of these cells.

Regarding steroid hormone receptor levels, no difference was demonstrated in endometrium from endometriotic vs. nonendometriotic women, and the levels remained low in endometriotic lesions without significant cyclic modulation. Endometriotic cells seem to be partially independent of hormonal regulation. This could explain why hormonal treatment is unable to eradicate endometriotic lesions.

The specific relationship between steroid hormones, apoptosis, and the development and maintenance of endometriosis remains unclear. It is well known that endometriotic cells respond to cyclic estrogen and P stimulation in an incomplete and variable fashion compared with endometrium. This could be due to differences in vascularity and the degree of surrounding fibrosis near the stroma. The absence of cyclic modulation of ER and PR in endometriotic lesions in our study was in agreement with previous observations.

There is no consensus concerning the levels of ER and PR expression in endometriosis, which were variable in different studies, either higher than in endometrium (35-36) or lower (37-38). The differences between the studies could be explained by the site of endometriotic biopsy (ovarian vs. peritoneal) or by the assay methods used.

In conclusion, for the first time, apoptosis, steroid hormone receptors, and cellular proliferation were evaluated simultaneously in endometrium from normal fertile patients (normal endometrium) and from patients with endometriosis (eutopic endometrium) and in peritoneal endometriosis. The induction of apoptosis seemed to be regulated by hormonal changes in the endometrium (normal and eutopic) and had an inverse correlation with the presence of bcl-2, as determined by immunohistochemistry. Endometrium from normal fertile patients and from endometriotic patients displayed similar pattern of parameters evaluated.

Conversely, the peritoneal endometriosis did not present cyclic variation in proliferation, steroid hormone receptor levels, and apoptotic markers (TUNEL, bcl-2) and thus had features different from those observed in the endometrium (normal and eutopic). Endometriotic cells are less sensitive to apoptosis. Altogether, these findings suggest that the success of ectopic implantation is unrelated to an excess of proliferation but rather is likely to be promoted by a decreased susceptibility to apoptosis.

Supported by grants from the Communauté Française de Belgique (Actions de Recherches Concertées), the Commission of European Communities, the Fonds de la Recherche Scientifique Médicale, the Fonds National de la Recherche Scientifique (FNRS, Belgium), the Fonds spéciaux de la Recherche (University of Liège), the Centre Anticancéreux près l'Université de Liège, FORTIS-Assurances, the Fondation Léon Frédéricq (University of Liège), the Direction Générale Des Technologies, De La Recherche Et De L’Energie (D.G.T.R.E.) from the "Région Wallonne," and the Fonds d'Investissements de la Recherche Scientifique (CHU, Liège, Belgium).

\section{References}

1. Sampson JA. Peritoneal endometriosis due to menstrual dissemination of endometrial tissue into the peritoneal cavity. Am J Obstet Gynecol 1927;14:422-69.

2. Jenkins S, Olive DL, Haney AF. Endometriosis: pathogenetic implications of the anatomic distribution. Obstet Gynecol 1986;67:335-8.

3. Stewart BW. Mechanisms of apoptosis: integration of genetic, biochemical, and cellular indicators. J Natl Cancer Inst 1994;86:1286-96.

4. Hopwood D, Levison DA. Atrophy and apoptosis in the cyclical human endometrium. J Pathol 1976;119:159-66. 
5. Keetel WC, Stein RJ. The viability of the cast-off menstrual endometrium. Am J Obstet Gynecol 1951;61:440-2.

6. Bartosik D, Jacobs SL, Kelly LJ. Endometrial tissue in peritoneal fluid. Fertil Steril 1986;46:796-800.

7. Barcz E, Kaminski P, Marianowski L. Role of cytokines in pathogenesis of endometriosis. Med Sci Monit 2000;6:1042-6.

8. Koninckx PR, Kennedy S, Barlow DH. Endometriotic disease: the role of the peritoneal fluid. Hum Reprod Update 1998;4:741-51.

9. Ruoslahti E, Reed JC. Anchorage dependence, integrins, and apoptosis. Cell 1994;77:477-8.

10. Frisch SM, Francis H. Disruption of epithelial cell-matrix interactions induces apoptosis. J Cell Biol 1994;124:619-26.

11. Stivala LA, Scovassi AI, Bianchi L, Prosperi E. Nuclear binding of cell cycle-related proteins: cyclin A versus proliferating cell nuclear antigen (PCNA). Biochimie 1995;77:888-92.

12. Noyes RW, Hertig AT, Rock J. Dating the endometrial biopsy. Fertil Steril 1950;1:3-25.

13. Beliard A, Noel A, Goffin F, Frankenne F, Foidart JM. Adhesion of endometrial cells labeled with (111)Indium-tropolonate to peritoneum: a novel in vitro model to study endometriosis. Fertil Steril 2003;79: 724-9.

14. Gebel HM, Braun DP, Tambur A, Frame D, Rana N, Dmowski WP. Spontaneous apoptosis of endometrial tissue is impaired in women with endometriosis. Fertil Steril 1998;69:1042-7.

15. Dmowski WP, Ding J, Shen J, Rana N, Fernandez BB, Braun DP. Apoptosis in endometrial glandular and stromal cells in women with and without endometriosis. Hum Reprod 2001;16: 1802-8.

16. Jones R, Searle R, Bulmer J. Apoptosis and bcl-2 expression in normal human endometrium, endometriosis and adenomyosis. Hum Reprod 1998;13:3496-502.

17. Watanabe H, Kanzaki H, Narukawa S, Inoue T, Katsuragawa H, Kaneko Y, et al. Bcl-2 and Fas expression in eutopic and ectopic human endometrium during the menstrual cycle in relation to endometrial cell apoptosis. Am J Obstet Gynecol 1997;176:360-8.

18. Suganuma N, Harada M, Furuhashi M, Nawa A, Kikkawa F. Apoptosis in human endometrial and endometriotic tissues. Horm Res 1997; 48(Suppl 3):42-7.

19. McLaren J, Prentice A, Charnock-Jones DS, Sharkey AM, Smith SK. Immunolocalization of the apoptosis regulating proteins Bcl-2 and Bax in human endometrium and isolated peritoneal fluid macrophages in endometriosis. Hum Reprod 1997;12: 146-52.

20. Harada M, Suganuma N, Furuhashi M, Nagasaka T, Nakashima N, Kikkawa F, et al. Detection of apoptosis in human endometriotic tissues. Mol Hum Reprod 1996;2:307-15.

21. Matsumoto Y, Iwasaka T, Yamasaki F, Sugimori H. Apoptosis and Ki-67 expression in adenomyotic lesions and in the corresponding eutopic endometrium. Obstet Gynecol 1999;94:71-7.

22. Nisolle M, Donnez J. Peritoneal endometriosis, ovarian endometriosis, and adenomyotic nodules of the rectovaginal septum are three different entities. Fertil Steril 1997;68:585-96.

23. Lessey BA, Castelbaum AJ, Sawin SW, Sun J. Integrins as markers of uterine receptivity in women with primary unexplained infertility. Fertil Steril 1995;63:535-42.

24. Gonzalez RR, Palomino A, Boric A, Vega M, Devoto L. A quantitative evaluation of alphal, alpha4, alphaV and beta3 endometrial integrins of fertile and unexplained infertile women during the menstrual cycle. A flow cytometric appraisal. Hum Reprod 1999;14:2485-92.

25. Tei C, Maruyama T, Kuji N, Miyazaki T, Mikami M, Yoshimura Y. Reduced expression of alphavbeta3 integrin in the endometrium of unexplained infertility patients with recurrent IVF-ET failures: improvement by danazol treatment. J Assist Reprod Genet 2003;20:13-20.

26. Nisolle M, Paindaveine B, Bourdon A, Berliere M, Casanas-Roux F, Donnez J. Histologic study of peritoneal endometriosis in infertile women. Fertil Steril 1990;53:984-8.

27. Balasch J, Creus M, Fabregues F, Carmona F, Ordi J, Martinez-Roman S, et al. Visible and non-visible endometriosis at laparoscopy in fertile and infertile women and in patients with chronic pelvic pain: a prospective study. Hum Reprod 1996;11:387-91.

28. Malik E, Berg C, Meyhofer-Malik A, Buchweitz O, Moubayed P, Diedrich K. Fluorescence diagnosis of endometriosis using 5aminole-vulinic acid. Surg Endosc 2000;14(5):452-5.

29. Nisolle M, Casanas-Roux F, Donnez J. Early-stage endometriosis: adhesion and growth of human menstrual endometrium in nude mice. Fertil Steril 2000;74:306-12. 
Published in: Fertility and Sterility (2004), vol.82, iss.1, pp. 80-85

Status: Postprint (Author's version)

30. Groothuis PG, Koks CA, de Goeij AF, Dunselman GA, Arends JW, Evers JL. Adhesion of human endometrial fragments to peritoneum in vitro. Fertil Steril 1999;71:1119-24.

31. Witz CA, Monotoya-Rodriguez IA, Schenken RS. Whole expiants of peritoneum and endometrium: a novel model of the early endometriosis lesion. Fertil Steril 1999;71:56-60.

32. Beliard A, Noel A, Goffin F, Frankenne F, Foidart JM. Role of endocrine status and cell type in adhesion of human endometrial cells to the peritoneum in nude mice. Fertil Steril 2002;78:973-8.

33. Liotta LA. Cancer cell invasion and metastasis. Sci Am 1992;266:54-59, 62-63.

34. Scotti S, Regidor PA, Schindler AE, Winterhager E. Reduced proliferation and cell adhesion in endometriosis. Mol Hum Reprod 2000;6: 610-7.

35. Lessey BA, Metzger DA, Haney AF, McCarty KS. Immunohistochem-ical analysis of estrogen and progesterone receptors in endometriosis: comparison with normal endometrium during the menstrual cycle and the effect of medical therapy. Fertil Steril 1989;51:40915.

36. Fujishita A, Nakane PK, Koji T, Masuzaki H, Chavez RO, Yamabe T, et al. Expression of estrogen and progesterone receptors in endometrium and peritoneal endometriosis: an immunohistochemical and in situ hybridization study. Fertil Steril 1997;67:856-64.

37. Howell RJ, Dowsett M, Edmonds DK. Oestrogen and progesterone receptors in endometriosis: heterogeneity of different sites. Hum Reprod 1994;9:1752-8.

38. Bergqvist A, Ferno M. Oestrogen and progesterone receptors in endometriotic tissue and endometrium: comparison of different cycle phases and ages. Hum Reprod 1993;8:2211-7. 